\title{
新模式下的核磁共振实验教学探索和提升
}

\author{
丁飞 1 , 张翠 2 , 邱晓航 $1,{ }^{*}$ \\ 1 南开大学化学学院, 化学国家级实验教学示范中心(南开大学), 天津 300071 \\ 2 南开大学材料科学与工程学院, 天津 300071
}

摘要: 核磁共振在化合物结构鉴定、动力学研究等方面具有重要的作用, 是一种非常重要的仪器分析手段, 也是大学仪 器分析实验课程的重要内容。借助于现代化教育手段, 可以拓展课前课后学习时间, 对核磁共振实验项目课堂教学进行 有效教学设计, 构建互联网时代的新型教学模式。

关键词: 核磁共振; 仪器分析实验; 有效教学

中图分类号: G64; O6

\section{Exploration and Improvement of NMR Experiment Teaching under New Form}

\author{
Fei Ding ${ }^{1}$, Cui Zhang ${ }^{2}$, Xiaohang Qiu 1,* \\ ${ }^{1}$ National Demonstration Center for Experimental Chemistry Education (Nankai University), College of Chemistry, \\ Nankai University, Tianjing 300071, China. \\ ${ }^{2}$ School of Materials Science and Engineering, Nankai University, Tianjin 300071, China.
}

\begin{abstract}
Nuclear Magnetic Resonance (NMR) plays an important role in the structure identification and kinetics research of compounds. It is an important instrumental analysis method, but also an indispensable content in the university instrumental analysis laboratory course. With the help of modern educational means, learning time has been expanded, an effective teaching design of NMR experiment project classroom teaching is carried out, and a new teaching mode in the Internet era is constructed.
\end{abstract}

Key Words: NMR; Instrumental analysis experiment; Effective teaching

核磁共振波谱仪是化学、生物学、药学、材料学等诸多学科必备的分析测试工具, 在化合物结 构鉴定、动力学研究等方面往往具有不可替代的作用。核磁共振波谱技术是高等院校化学实验教学 的重要内容, 在各种层次的实验项目中扮演了重要角色 ${ }^{[1-7]}$, 但由于仪器昂贵、技术更新等原因, 实 验教学中多采取做样品、取数据的模式, 学生不能真正掌握波谱技术, 对仪器也很难有深入的了解。 随着互联网技术的进步, 现代教学手段为实验教学注入了新的活力, 为改善实验教学效果提供了技 术保障。南开大学化学学院自2002年起使用超导脉冲傅里叶变换核磁(Varian Mercury Vx-300)在本科 生仪器分析实验中开设核磁共振波谱实验项目, 让本科阶段的学生有机会接触到最先进的核磁共振

收稿: 2020-12-30; 录用: 2021-01-12; 网络发表: 2021-01-21

*通讯作者, Email: qiuxh@nankai.edu.cn

基金资助: 2020 年南开大学教学改革项目(NKJG2020335) 
技术, 近年来又通过更新实验内容、引入信息技术手段、增加多元化学习等有效教学方法对实验项 目进行了优化, 提高了教学要求, 教学效果得到提升。

\section{1 传统教学中存在的问题}

\section{1 实验教学模式造成的问题。}

核磁共振波谱是仪器分析课程中的一个实验项目。在仪器分析课程中, 基于大型仪器的特点, 采取学生循环分组的形式进行教学, 每节课一组共四名学生, 教学时长 4 学时。这种模式造成的直接 后果是理论课与实验课不同步, 很多学生在进行实验时, 缺乏基础知识铺垫, 导致理解困难。所以 很多仪器分析课程中, 教师在课上进行理论讲解, 占用了较多课时, 大大限制了学生的操作时间。 另一方面, 学生在短时间内很难完全吸收并掌握实验教师的指导内容, 实验后又缺乏继续讨论的平 台, 实验过后无法留下深刻印象。长期的结果是学生不重视仪器分析实验, 做实验走马观花, 按照指 示简单完成操作, 只求实验数据; 对仪器原理掌握不牢, 不能建立理论与实际应用的关联; 更谈不 上用课堂知识解决实际问题。而授课教师面对不同组别的学生, 多次重复授课内容, 容易产生倦急 感, 影响教学效果。

\section{2 由仪器造成的问题}

核磁共振技术涉及物理、数学、化学等多学科的众多知识点, 自 20 世纪70年代以来经过多次技 术升级, 如引入高磁感应强度的磁体, 使用计算机技术对数据进行傅里叶变换, 探头技术也在不停 革新, 出现了众多功能各异的探头, 学习难度和深度远超一般的分析仪器。另外作为大型仪器, 价值 昂贵数量少, 每组学生只能轮流操作, 本不充足的课时更加捉襟见肘; 大型仪器非常精密, 部分零 部件较易损坏, 对学生开放的操作较少 ${ }^{[8-13]}$ 。

\section{2 基于教育信息化技术的课程设计}

我国《教育信息化十年发展规划(2011-2020年)》指出: “教育信息化的发展要以优质的教育资源 和信息化学习环境建设为基础, 以教育理念的创新为先导, 以教育模式和学习方式的创新为核心”。

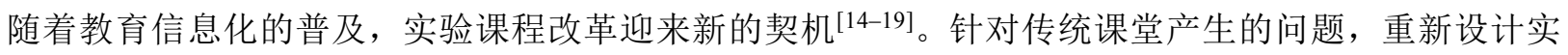
验项目, 探索网络时代教学的新模式。

\section{1 准确定位教学目标, 精心组织教学内容}

核磁共振波谱实验面向化学专业高年级学生开设, 通过该项目学习, 学生应掌握核磁共振原理, 初步掌握核磁共振波谱仪的使用, 并能正确解析波谱信息。此外, 学生还能通过了解仪器的更新过 程体会到科技创新的趋势, 建立创新学习、终身学习的理念。在与理论课任课教师沟通和对学生进 行调研的基础上, 发现理论课讲解偏重基本理论和应用, 学生可以较好地掌握屏蔽常数、化学位移、 偶合等基础知识, 但对与超导核磁技术关系紧密的采样过程、脉冲相关知识、异核耦合、驰豫等概 念理解不深, 如気代试剂残余氢的出峰由于涉及氞-氢的耦合, 裂分并不符合 $n+1$ 规则, $90^{\circ}$ 脉冲及 脉冲序列的概念理论课较少涉及, 学生普遍反映不太了解。对核磁共振实验教学中重要的知识点进 行梳理后(图1), 在实验知识点设计方面注意与理论课无缝对接, 既弥补基本理论的不足, 又侧重对 超导核磁共振技术的理解, 同时各个环节相互渗透, 前后呼应, 使教学各环节形成有机的整体。在 原有教学内容的基础上, 补充了科学前沿应用, 保证实验有足够 “营养”。

\section{2 合理规划教学过程, 提高课程挑战度}

实验教学分为课前预习、课上理论讲解、实验操作、翻转教学和课后讨论反馈五个环节。利用 信息技术将预习内容图文并茂地呈现出来, 采取线上课堂的形式布置给学生, 要求学生在课前做好 实验前的知识准备。这相当于把学生的学习时间拓展到课前, 无疑提高了学生的学习难度, 却可保 证课上有足够多的操作时间, 改善学生的操作体验。课堂教学中, 因为学生预习效果好, 可采取翻 转课堂的方式激发学生的主动性, 课后讨论也更有针对性和实效性。 


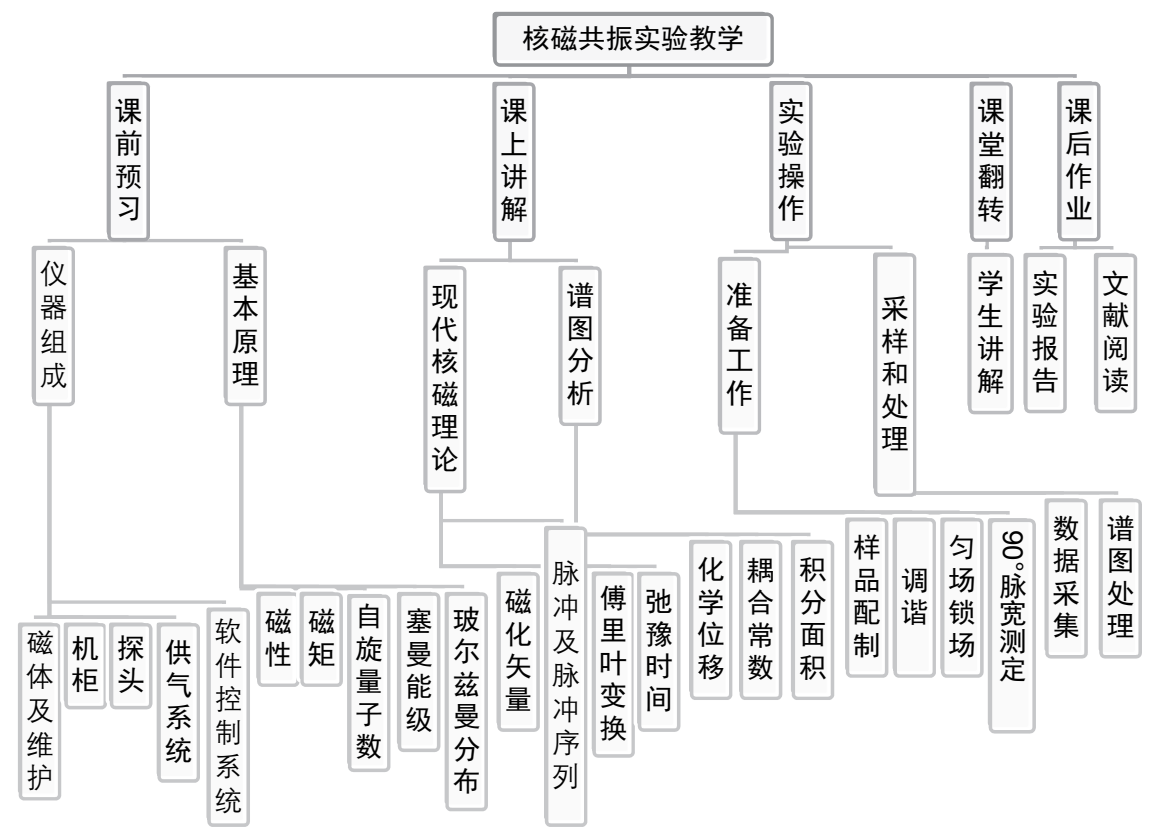

图1＼cjkstart核磁共振实验教学流程图及主要知识点

\section{3 充分运用信息技术，增强课程创新性}

信息技术为实验预习提供了平台。传统教学模式中, 很多学生在预习时, 只是机械抄写实验讲 义, 达不到预习的效果。我们将预习资料做成图文并茂的PPT课件, 还配有语音讲解和录制的实验小 视频, 这些材料上传至课程在线平台, 辅助微信群等社交软件答疑解惑, 学生可以充分完成课前准 备。

课堂教学也不再是教师灌输式讲解。课堂采取雨课堂等教学工具检查学生的预习效果, 做好查 漏补缺。对预习中共性问题给予重点解读, 提高了课堂讲解的针对性。

\section{4 翻转课堂教学模式, 体现课程高阶性}

在课堂讲解和实验操作结束后, 学生结合充分的预习对仪器有了较为深入的理解, 此时利用课 堂结束前的时间进行课堂翻转, 进行讨论反馈, 可以起到更好的作用。此时抛出科研中的实例, 让 学生分析讨论, 一方面检验学习效果, 同时可以充分调动学生的学习热情, 激发学生持续学习的欲望。

\section{3 互联网新模式的课程实践}

\section{1 考核预习效果, 提高预习效率}

为保证预习效果, 在预习材料中包含习题与测试。课件的预习和习题的完成体现为部分实验课 成绩。这种方式可以吸引学生自主学习, 提高预习质量; 同时督促学生重视实验预习环节, 为课堂 教学做好充足准备。学生在预习过程中产生的疑问可以随时通过社交软件提出, 由任课教师及时进 行解答, 原本点对点的单线答疑方式变更为点对面的多线方式, 一个疑问的解答可以惠及整个课程 群中所有的学生, 其他同学在提问获得解答的过程中也能加深对相关知识点的理解, 带动整体预习 水平的提高。

\section{2 课上讲解因材施教, 增强教学创造性}

有了好的预习效果, 有效节省了课堂讲解时间。课上教师可以根据不同学生的预习情况, 有针 对性地进行知识讲解, 对学生提出的问题进行个性化讨论。教师可以有的放矢地把知识点加深扩展, 细节讲好讲透。例如图1中现代核磁理论的傅里叶变换、脉冲及脉冲序列、弛豫时间及其应用等内容, 这些知识有一定的深度和难度, 在原来的讲解过程中基本不会涉及, 或者只是简单介绍, 远远不能 
满足学生对现代核磁共振技术深层次的学习需求。在学生充分预习, 且课堂时间充裕的情况下, 授 课教师需要根据学生的个性化反馈, 列举合适的例子加以说明, 做到因材施教, 发挥的空间较大。 由于避免了重复性讲解, 教师的创造性也得到激发, 保持了授课热情。

\section{3 操作训练以人为本, 激发学习主动性}

作为一门实验类课程, 一个重要环节是学生动手实操, 这也是学生最为感兴趣的部分。尤其是 对核磁共振这种大型仪器, 价格昂贵, 大部分本科生日常很难有机会接触。在新模式下, 学生获得 了更多的实验操作时间，因此实验操作部分可以进一步充实。

传统模式的核磁共振波谱实验中, 由于时间限制, 由学生进行的操作只有使用 “自动匀场” 和 “自动锁场” 功能将磁场调节均匀, 随后进行数据的采集和处理。这样操作简单乏味, 对学生毫无 吸引力, 也无法体现获得核磁共振波谱的全过程。新模式下, 我们将调谐、 $90^{\circ}$ 脉宽测定等超导核磁 日常维护的基本操作纳入学生操作范围, 每组四名学生轮流进行实验操作, 均包括进样、自动匀场 锁场、 $90^{\circ}$ 脉宽测定、数据采集、谱图处理打印等部分。自动调谐过程较短, 且过多的调谐次数可能 损坏探头中的电机, 经与工程师沟通后确认可以承受每次实验课有一次调谐操作, 因此该环节只由 第一名同学操作并演示。9 $0^{\circ}$ 脉宽测定虽对实验结果没有直接影响, 但对理解磁化矢量和脉冲序列有 非常好的作用。学生通过实际操作, 全面了解做好一个核磁共振谱图所必须的一些基本流程, 同时 对其中蕴含的共振频率、磁化矢量、脉冲序列等概念有更加深入的了解, 理清 “实验操作” 与 “仪 器原理”之间的关系, 充分发挥出实验教学的作用。

经过调整后, 4 个学时的实验课堂时间分配发生了积极的变化, 讲授部分由 90 分钟缩减到了一个 小时以内, 学生实验操作部分由不到一小时扩展为90分钟左右, 操作的同时授课教师对采样时间、 累加次数、脉冲间隔、谱宽设置、中心频率、增益调整、匀场算法等参数进行讲解, 同时引导学生 修改部分参数进行体验，更加贴近实战。剩余约40分钟的时间供师生交流。

\section{4 课堂翻转即时评测, 教学效果有效延伸}

学生实际操作之后, 对于相关知识已经有了具体感性的认识。在此基础上, 采取课堂翻转的形 式让学生走上讲台, 学生讲解核磁共振信号产生的基本过程, 画出磁化矢量翻转角度和出峰的对应 关系, 或针对一些关键知识点提出问题, 学生互相讨论并进行解答, 如分析氛代丙酮在氢谱和碳谱 中溶剂峰的裂分; 讨论弛豫时间测定实验中磁化矢量的变化; 实际测试中一维实验普遍采用 $30^{\circ}$ 或 $45^{\circ}$ 脉宽激发样品, 而实验中增加了 $90^{\circ}$ 脉宽测定内容, 分析讨论 $90^{\circ}$ 脉宽对不同实验的影响, 为何引入 该部分操作; 选择不同脉宽时考虑脉冲间隔时间的修改以保证定量准确性; 讨论各类核磁共振实验 需要关注的重要参数等, 进一步调动学生的情绪, 锻炼学生的团结协作和表达能力, 对所学知识进 行总结和梳理, 深化课堂教学效果, 即时进行巩固。

\section{5 实验报告总结反思, 结合科研深度学习}

实验课程的最后一个环节是整理完成实验报告。为了提高学习的高阶性, 除了根据实验所得的 样品氢谱进行化合物含量的计算, 同时把样品的碳谱数据交给学生, 要求学生结合碳谱数据对化合 物进行完全的归属, 并练习用规范的科研写作方式对实验结果进行表述。通过对化合物氢谱和碳谱 的比较, 学生对耗时长、操作更复杂的碳谱也有了初步的了解。为了进一步加深学生对核磁共振波 谱仪的认识, 在课程平台上提供核磁共振在实际科研工作中的运用实例, 供学生课后深度学习, 达 到锻炼科学思维、理论联系实际、学以致用的目的。

\section{4 结语}

互联网改变着人们的生活, 也改变着高等教育的模式。在信息技术的支持下, 实验教学的过程 可以更加合理, 课程内容更加充实, 课堂氛围更加活跃。学生的学习不再局限于规定课时内, 学生 必须付出更多的精力, 学到的知识也更加牢固。新模式提高了课程的挑战度, 却满足了学生对新技 术、大仪器的好奇心, 带给他们深度学习的获得感和成就感, 学习效果有了明显提升。 
[1] 刘硕, 王晓静, 韩杰. 化学教育, 2017, 38 (6), 24.

[2] 林沛和. 实验室研究与探索, 2005, 24 (6), 76.

[3] 周格, 白怀勇, 王殿生. 实验室研究与探索, 2013, 32 (8), 47.

[4] 张慧，曹卫国，童玮琦. 实验技术与管理, 2009, 26 (6), 124

[5] 李春远, 卢其明, 陈敏. 实验技术与管理, 2015, 32 (1), 11.

[6] 丁唯嘉, 祝钧杰, 陈敏. 实验技术与管理, 2017, 34 (3), 47.

[7] 梁向晖, 毛秋平, 牛君涛. 实验技术与管理, 2019, 36 (1), 76.

[8] 张万群, 邵伟, 柯玉萍. 化学教育, 2018, 39 (1), 24.

[9] 冯国栋, 宋志光. 广州化工, 2017, 45 (7), 137.

[10] 周立敏, 杨桂朋, 高先池. 实验科学与技术, 2016, 14 (1), 148.

[11] 李爱峰, 孙爱玲, 柳仁民. 大学化学, 2012, 27 (5), 26.

[12] 马祥英, 陈其锋, 许海棠. 实验科学与技术, 2013, 11 (2), 76.

[13] 王聪, 王远红, 王义. 实验技术与管理, 2016, 33 (7), 160.

[14] 邓小娟, 丁国生, 陈小平. 分析测试技术与仪器, 2017, 23 (3), 139.

[15] 郭婷, 梁立, 郑奕娜. 广东化工, 2018, 45 (6), 229.

[16] 李中峰, 王英锋, 李凯. 首都师范大学学报(自然科学版), 2011, 32 (化学专辑), 38 .

[17] 毛秋平, 梁向晖, 钟伟强. 广州化工, 2015, 43 (7), 154.

[18] 王顷, 李荣, 姜子涛. 教育现代化, 2019, No. 83, 56.

[19] 郑超，陈润锋，周广荣. 实验技术与管理, 2011, 28 (10), 130. 